\title{
ESTUDO DAS COBERTURAS SUPERFICIAIS HOLOCÊNICAS NA SUPERFÍCIE DE URUCAIA NA BACIA HIDROGRÁFICA DO RIO CORUMBATAÍ-SP
}

\author{
Renê Lepiani Dias ${ }^{(a)}$, Archimedes Perez Filho ${ }^{(b)}$ \\ (a) Instituto Federal de Educação, Ciência e Tecnologia do Sul de Minas Gerais - Campus Muzambinho, \\ rene.dias@muz.ifsuldeminas.edu.br \\ (b) Departamento de Geografia/Instituto de Geociências, Universidade Estadual de Campinas, archi@ige.unicamp.br
}

\section{Eixo: 5.GEOCRONOLOGIA E ESTUDOS PALEOAMBIENTAIS}

\begin{abstract}
Resumo
O objetivo deste trabalho constitui-se na análise geocronológica das coberturas superficiais holocênicas depositadas sobre a Superfície de Urucaia, na bacia hidrográfica do rio Corumbataí-SP. Para atingir tal meta, adotou-se como procedimento metodológico a seleção de seis pontos para coleta das coberturas superficiais no nível de aplainamento identificado a 1 metro de profundidade. Resultados obtidos pela análise granulométrica, interpretados por meio do diagrama textural, permitiram classificar os sedimentos em relação a sua textura. Com auxilio da morfoscopia, identificou-se o agente de transporte responsável pela deposição dos sedimentos. Por meio de datação absoluta por Luminescência Opticamente Estimulada (LOE) foi possível determinar o momento de deposição das mesmas, auxiliando na discussão no contexto de pulsos climáticos ocorridos durante o final do Quaternário no Estado de São Paulo. Conclui-se que houve a ocorrência de oscilações climáticas mais secas que o clima atual durante o Holoceno, responsáveis pela deposição das coberturas superficiais depositadas sobre a Superfície de Urucaia, desde 5.200 anos A.P.
\end{abstract}

Palavras chave: Superfície de Urucaia; LOE; coberturas superficiais.

\section{Introdução}

O estudo das coberturas superficiais holocênicas localizadas sobre a Superfície de Urucaia na bacia hidrográfica do rio Corumbataí-SP tem como finalidade compreender a espacialização das mesmas, assim como os processos de transporte e erosão, no contexto das oscilações climáticas quaternárias.

O presente estudo leva em consideração a hipótese de que as coberturas superficiais depositadas sobre a Superfície de Urucaia possam estar relacionadas às oscilações climáticas ao longo do Quaternário, sendo que a compreensão da formação e evolução das mesmas torna-se significativa para análise das mudanças ambientais ocorridas na escala recente do tempo da natureza.

Nesta perspectiva, o objetivo deste estudo constitui-se no estudo geocronológico das coberturas superficiais holocênicas, caracterizadas pelo material de origem dos solos, a 1,0 metro de profundidade, depositadas sobre a Superfície de Urucaia na bacia hidrográfica do rio Corumbataí-SP. 


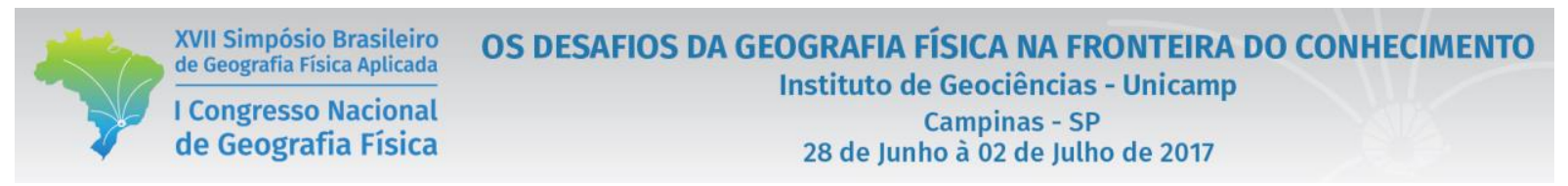

Logo, o estudo das coberturas superficiais holocênicas depositadas sobre a Superfície de Urucaia tem como finalidade fornecer levantamento detalhado e descrição explicativa das formas de relevo associadas a sua evolução, e, além disto, analisar os mecanismos que atuaram na esculturação e deposição das mesmas, associadas à ocorrência de oscilações climáticas.

\section{2. Área de estudo}

A bacia hidrográfica do rio Corumbataí foi desenvolvida a partir do Cenozoico, as quais cabeceiras de drenagem localizam-se nas Cuestas da Serra Geral, em litologias típicas da bacia sedimentar do Paraná. A altimetria varia entre 1068 m na Serra do Cuscuzeiro, na região de Analândia, limite dos divisores topográficos, e a $470 \mathrm{~m}$ na desembocadura do rio Piracicaba, na cidade homônima.

O resultado da ação dos processos morfogenéticos através do tempo está impresso nas grandes linhas e nas formas de detalhe do relevo representadas nos diferentes níveis de aplainamento que se destacam na paisagem regional.

A bacia hidrográfica do rio Corumbataí, localizada no setor centro-ocidental da Depressão Periférica Paulista (Figura 1), é caracterizada pela presença de níveis de aplainamento neogênicos (PENTEADO, 1968).

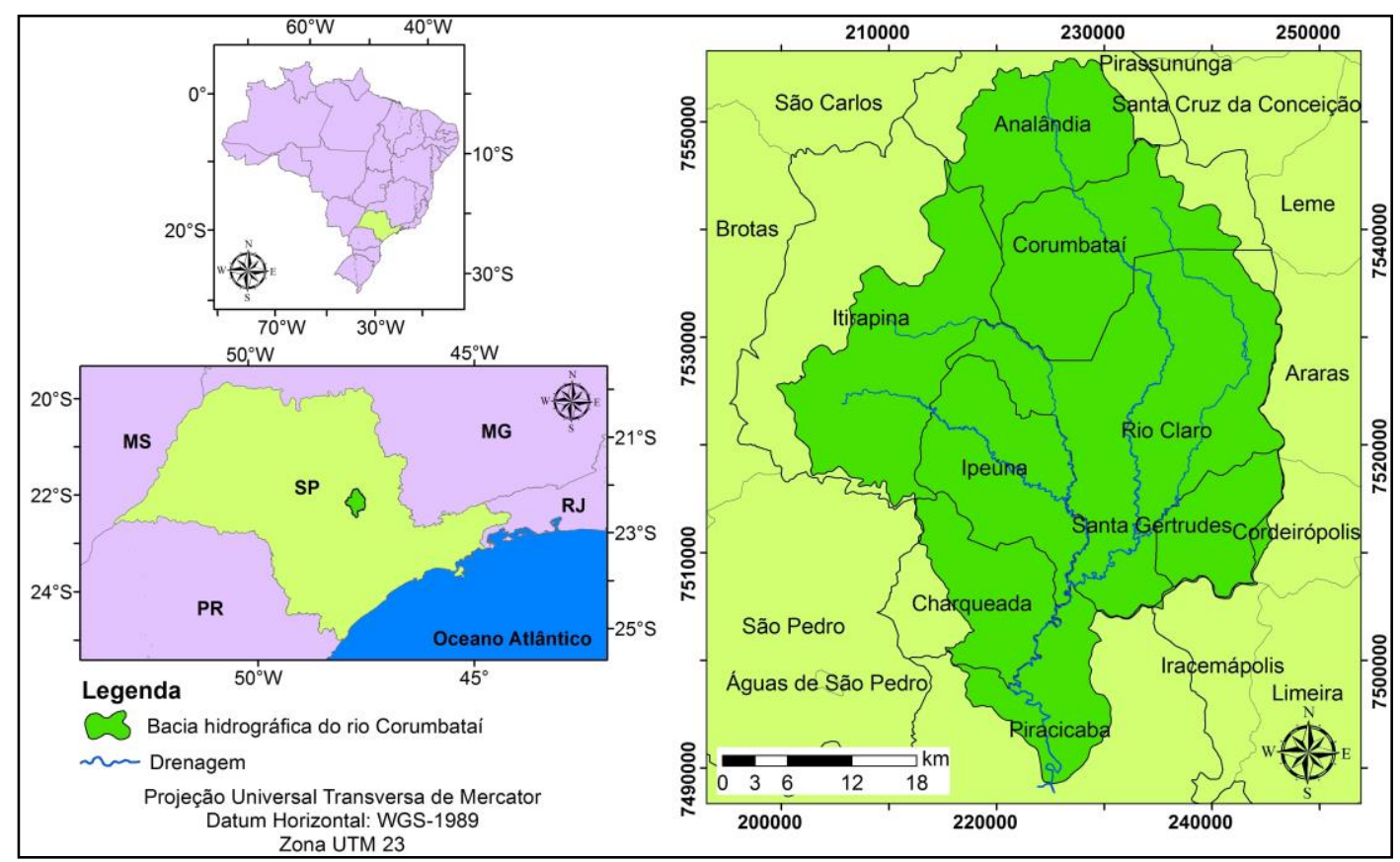

Figura 1 - Localização da área de estudo 
Para Melo e Ponçano (1983), devido à atuação endógena e exógena durante o Pleistoceno e Holoceno, a elaboração da paisagem na bacia hidrográfica do rio Corumbataí, refletem interação de fatores litológicos, tectônicos e climáticos, igualmente importantes.

Datada do Plioceno, a Superfície de Urucaia é a mais antiga das superfícies interplanálticas elaborada em clima seco. Fruto de grande fase de pediplanação, seus vestígios nivelam topos das colinas sedimentares em torno de 700 a 750 metros, posicionada em todo setor limítrofe, na área de transição das Cuestas Basálticas e da Depressão Periférica Paulista, esculpida nos arenitos Botucatu e Piramboia, nos interflúvios adjacentes ao relevo de cuestas e em rochas básicas intrusivas (TAVARES et al., 2007).

Verifica-se que a Superfície de Urucaia é recoberta por coberturas superficiais, representadas pela unidade sedimentar das coberturas não diferenciadas neocenozoicas, constituídas por materiais bastante arenosos, sem estruturas sedimentares, depositados no contato com blocos de cangas lateríticas autóctones, e/ou tênues crostas de arenitos limonitizados, que resultam no material de origem dos solos.

\section{Procedimentos metodológicos}

O recurso metodológico adotado como condutor deste trabalho está pautado na abordagem sistêmica, realizada a partir da investigação das alterações do sistema morfoclimático na compreensão da dinâmica de evolução da paisagem ao longo do Quaternário.

Para atingir o objetivo, foram identificados e selecionados seis pontos de interesse em trabalho de campo, onde foram coletadas amostras para realização de análises laboratoriais: granulometria, morfoscopia e datação absoluta por LOE (Figura 2) (Tabela 1).

A análise granulométrica foi realizada pelo método do densímetro (EMBRAPA, 1997), baseado na sedimentação das partículas que compõem o solo, para obter a classe textural das amostras coletadas.

A morfoscopia foi realizada com intuito de identificar e interpretar a origem e o agente de transporte das coberturas superficiais, baseados na relação entre arredondamento, esfericidade e grau de seleção dos sedimentos, com base na tabela de comparação visual proposta por Compton (1962). Para realização desta análise foram utilizadas três amostras da análise granulométrica, selecionando as classes areia grossa $(0,50$ $\mathrm{mm})$, areia média $(0,25 \mathrm{~mm})$ e areia fina $(0,125 \mathrm{~mm})$, analisando-se 300 grãos de cada amostra (100 grãos de cada classe textural).

Com auxílio do mapeamento de solos semidetalhado, escala 1:100.000 (Folha São Carlos - IAC, 1981), e trabalhos de campo foi identificado o tipo de solo predominante dos pontos coletados. 


\section{OS DESAFIOS DA GEOGRAFIA FÍSICA NA FRONTEIRA DO CONHECIMENTO Instituto de Geociências - Unicamp Campinas - SP \\ 28 de Junho à 02 de Julho de 2017}

Tabela 1 - Localização das coberturas superficiais holocênicas

\begin{tabular}{c|c|c|c} 
Ponto de Coleta & Localização Geográfica & $\begin{array}{c}\text { Altimetria } \\
(\mathbf{m})\end{array}$ & $\begin{array}{c}\text { Profundidade } \\
\text { da coleta (m) }\end{array}$ \\
\hline Amostra 1 & $22^{\circ} 07^{\prime} 19^{\prime \prime} \mathrm{S}-47^{\circ} 38^{\prime} 18^{\prime \prime} \mathrm{W}$ & 750 & 1,0 \\
\hline Amostra 2 & $22^{\circ} 07^{\prime} 49^{\prime \prime} \mathrm{S}-47^{\circ} 38^{\prime} 41^{\prime \prime} \mathrm{W}$ & 705 & 1,0 \\
\hline Amostra 3 & $22^{\circ} 17^{\prime} 54^{\prime \prime} \mathrm{S}-47^{\circ} 48^{\prime} 14^{\prime \prime} \mathrm{W}$ & 727 & 1,0 \\
\hline Amostra 4 & $22^{\circ} 16^{\prime} 30^{\prime \prime} \mathrm{S}-47^{\circ} 39^{\prime} 37^{\prime \prime} \mathrm{W}$ & 714 & 1,0 \\
\hline Amostra 5 & $22^{\circ} 18^{\prime} 21^{\prime \prime} \mathrm{S}-47^{\circ} 37^{\prime} 27^{\prime \prime} \mathrm{W}$ & 736 & 1,0 \\
\hline Amostra 6 & $22^{\circ} 19^{\prime} 59^{\prime \prime} \mathrm{S}-47^{\circ} 45^{\prime} 33^{\prime \prime} \mathrm{W}$ & 726 & 1,0 \\
\hline
\end{tabular}

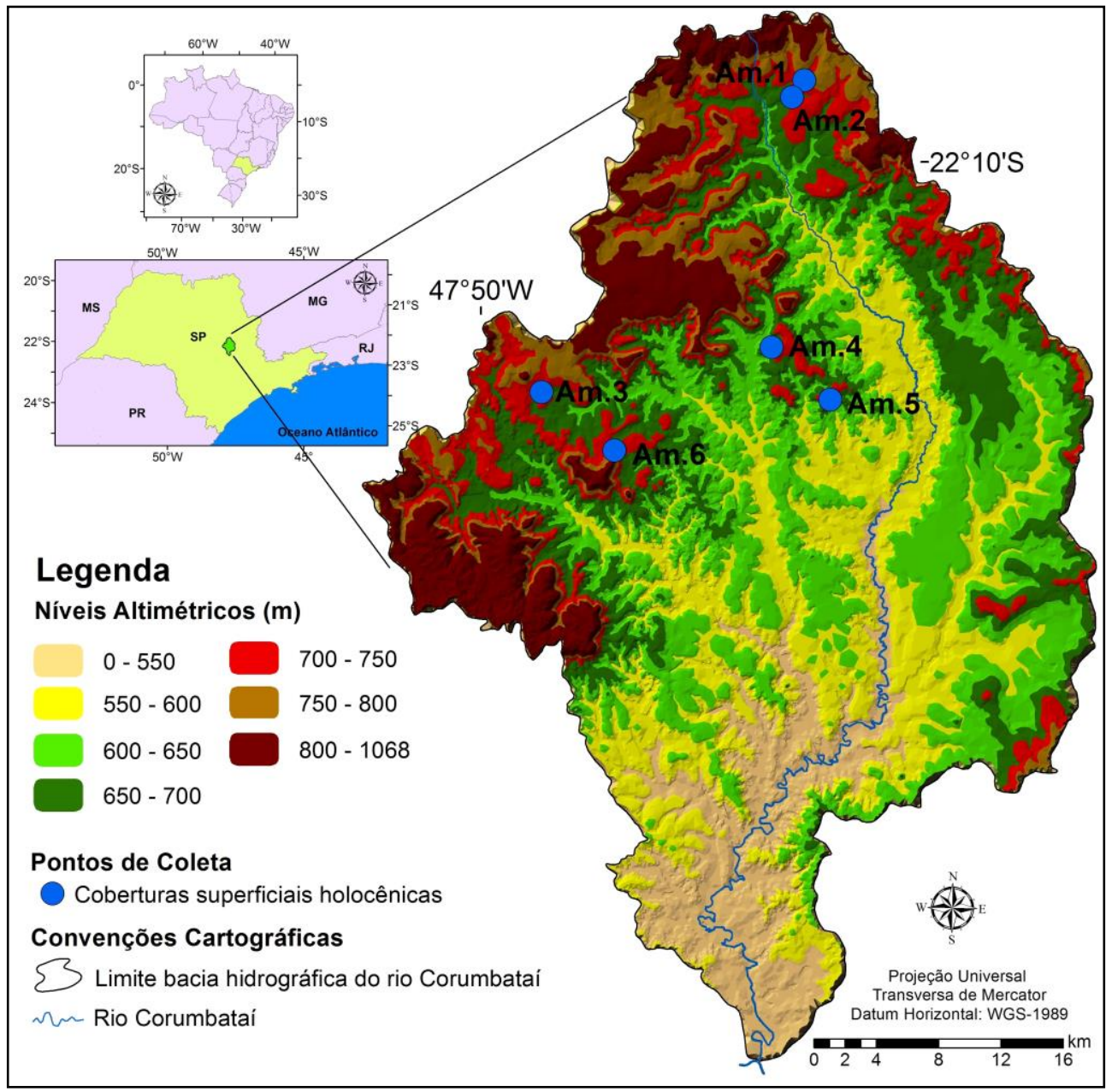

Figura 2 - Localização das amostras coletadas

Datações absolutas por LOE foram realizadas, utilizando-se do método SAR (Single Aliquot Regenerative-dose) em grãos de quartzo, proposto por Wintle e Murray (2006), com 15 alíquotas, para determinar o momento de deposição das coberturas superficiais. 


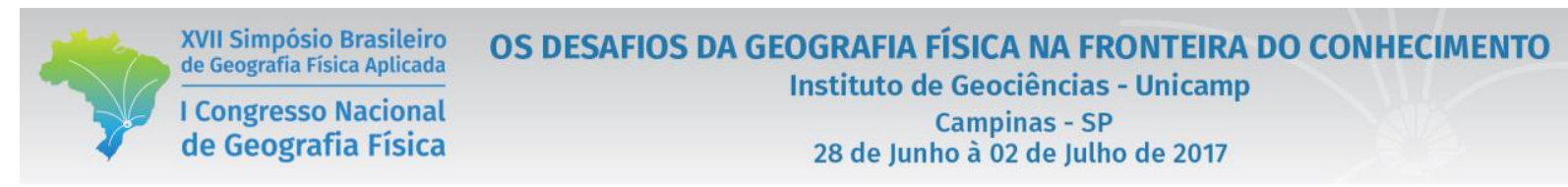

Para Duller (2004), datações absolutas por meio da luminescência de minerais constituem um dos únicos métodos geocronológicos de determinação da ocorrência de eventos geológicos, que podem ser relacionados às idades de deposição de sedimentos, principalmente de depósitos quaternários, já que a técnica tem abrangência temporal desde cerca de 100 anos A.P. até 1 Ma A.P., dependendo dos níveis de saturação do material analisado.

Segundo Côrrea (2001), a idade de sedimentação é determinada ao se considerar que a luminescência nos minerais naturais é uma função da exposição à radiação ambiental, e que pode ser esvaziada por exposição à luz durante transporte e deposição. Para o autor, métodos de datação por luminescência são capazes de estabelecer o período de tempo transcorrido desde que a população aprisionada de elétrons nos minerais cristalinos (quartzo ou feldspato) foi liberada pela última vez, já que abrangem uma gama de técnicas baseadas no acúmulo de cargas radioativas produzidas por esta população.

\section{Resultados e discussões}

As coberturas superficiais da Superfície de Urucaia estão localizadas altimetricamente entre 700-750 metros, predominantemente no alto curso da bacia hidrográfica, cujo material de recobrimento está localizado sobre topos dos antigos pedimentos, com textura bastante arenosa, com mais de $85 \%$ de areia, sendo constituída em maior proporção por areia média e fina (Tabela 2).

Tabela 2 - Análise granulométrica das coberturas superficiais holocênicas

\begin{tabular}{|c|c|c|c|c|c|c|c|c|}
\hline \multirow[b]{2}{*}{ Amostra } & \multicolumn{6}{|c|}{ Areia (\%) } & \multirow{2}{*}{$\begin{array}{c}\text { Argila } \\
(\%)\end{array}$} & \multirow{2}{*}{$\begin{array}{c}\text { Silte } \\
(\%)\end{array}$} \\
\hline & $\begin{array}{l}\text { Muito } \\
\text { Grossa }\end{array}$ & Grossa & Média & Fina & $\begin{array}{c}\text { Muito } \\
\text { Fina }\end{array}$ & TOTAL & & \\
\hline Amostra 1 & 0,4 & 2,1 & 42,3 & 36,4 & 13,0 & 94,2 & 4,6 & 1,2 \\
\hline Amostra 2 & 0,4 & 1,8 & 21,3 & 49,0 & 12,0 & 84,5 & 11,8 & 3,7 \\
\hline Amostra 3 & 1,4 & 6,7 & 26,9 & 39,3 & 12,3 & 86,6 & 10,9 & 2,5 \\
\hline Amostra 4 & 0,0 & 0,0 & 5,2 & 61,8 & 16,6 & 83,6 & 11,4 & 5,0 \\
\hline Amostra 5 & 0,0 & 0,0 & 7,0 & 58,4 & 23,9 & 89,3 & 7,4 & 3,3 \\
\hline Amostra 6 & 0,0 & 2,4 & 21,7 & 41,7 & 21,8 & 87,6 & 8,9 & 3,5 \\
\hline
\end{tabular}

A partir desta cobertura superficial ocorre o desenvolvimento de Neossolos Quartzarênicos associados às coberturas não diferenciadas cenozoicas, assentadas sobre camada de concreções ferruginosas autóctones. 
XVII Simpósio Brasileiro

de Geografia Física Aplicada

I Congresso Nacional

de Geografia Física
OS DESAFIOS DA GEOGRAFIA FÍSICA NA FRONTEIRA DO CONHECIMENTO

Instituto de Geociências - Unicamp

Campinas - SP

28 de Junho à 02 de Julho de 2017

Resultados da análise morfoscópica apontaram que os grãos de areia das coberturas superficiais eram bem selecionados, e apresentavam alta esfericidade, sendo mais arredondados, com bordas suavizadas e aparência fosca (Figura 3).

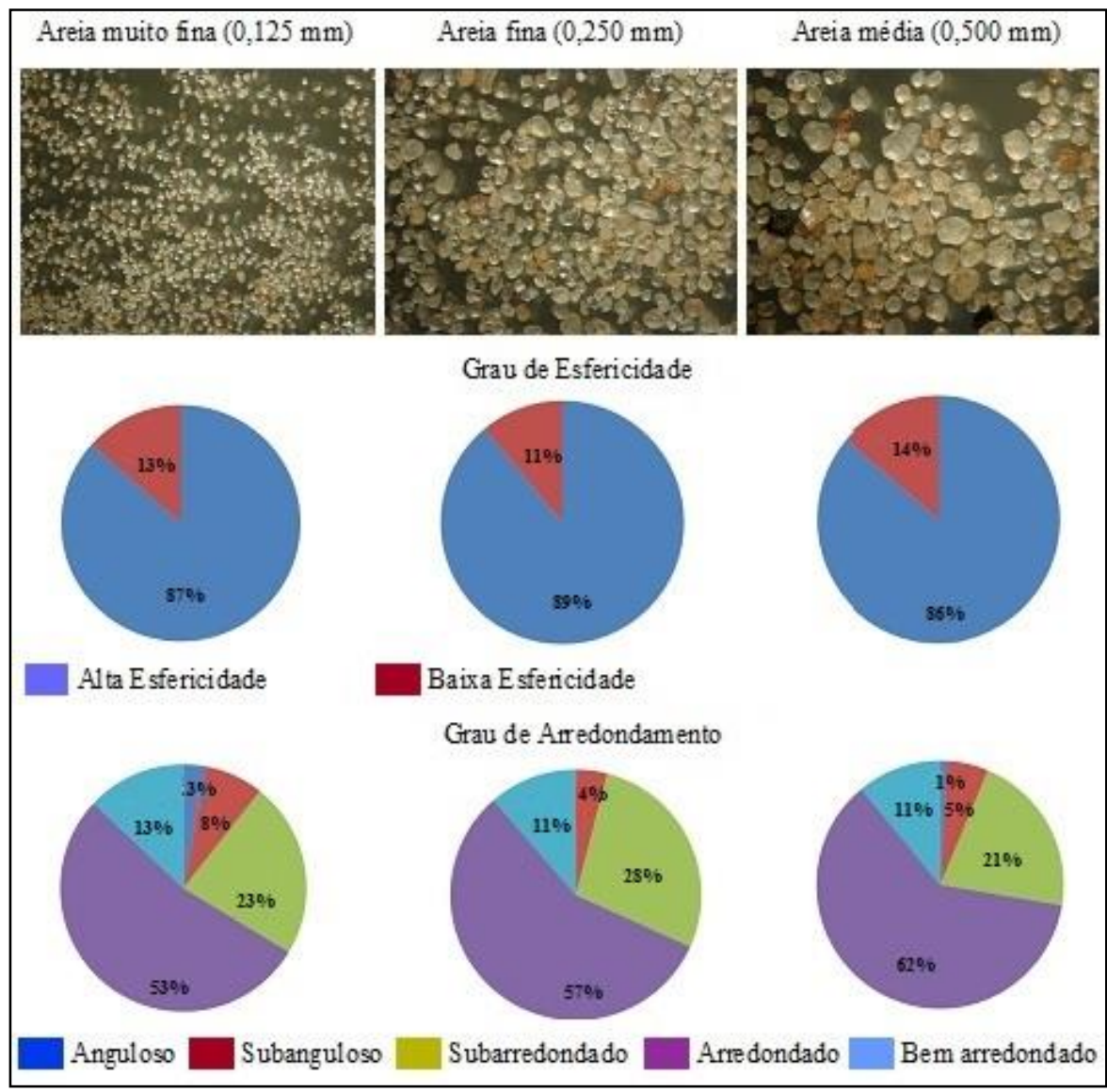

Figura 3 - Análise morfoscópica

Os limites topográficos da área de estudo são representados pela Serra do Cuscuzeiro (Morro do Cuscuzeiro e Pedra do Camelo como formas de relevo significativas), localizadas ao norte da bacia hidrográfica, na região de Analândia, e a Serra de Santana, localizada a nordeste na região de Itirapina. Estas áreas são formadas geologicamente pelos arenitos eólicos da Formação Botucatu, provável área fonte dos sedimentos depositados sobre a Superfície de Urucaia, ao longo do Pleistoceno, posteriormente após a definição do nível neogênico I.

Mineralogicamente, os grãos de areia das coberturas superficiais da Superfície de Urucaia apresentaram características semelhantes aos grãos de quartzo dos arenitos da Formação Botucatu, conforme descrição 


\section{OS DESAFIOS DA GEOGRAFIA FÍSICA NA FRONTEIRA DO CONHECIMENTO \\ Instituto de Geociências - Unicamp \\ Campinas - SP \\ 28 de Junho à 02 de Julho de 2017}

apresentada por Penteado (1968) e Björnberg (1965), o que contribui com a hipótese de que estas coberturas superficiais sejam provenientes do seu intemperismo.

Por meio da datação absoluta por LOE das amostras coletadas foi possível determinar o momento de deposição das coberturas superficiais holocências que recobrem a Superfície de Urucaia (Tabela 3).

Tabela 3 - Datação absoluta por LOE das coberturas superficiais

\begin{tabular}{c|c|c|c|c|c|c|c|c} 
Amostra & $\begin{array}{c}\text { Dose Anual } \\
(\boldsymbol{\mu G y} / \mathbf{a n o})\end{array}$ & $\begin{array}{c}\mathbf{P} \\
(\mathbf{G y})\end{array}$ & $\begin{array}{c}\text { Desvio } \\
\text { Padrão }\end{array}$ & $\mathbf{T h}(\mathbf{p p m})$ & $\mathbf{U}(\mathbf{p p m})$ & $\mathbf{K}(\boldsymbol{\%})$ & $\begin{array}{c}\text { Umidade } \\
(\boldsymbol{\%})\end{array}$ & $\begin{array}{c}\text { Idade } \\
(\mathbf{a n o s} \text { A.P.) }\end{array}$ \\
\hline Amostra 1 & $930 \pm 90$ & 2,6 & 1,0 & $2,641 \pm 0,095$ & $0,348 \pm 0,089$ & $0,440 \pm 0,064$ & 2,9 & $2.800 \pm 430$ \\
\hline Amostra 2 & $1.355 \pm 125$ & 4,1 & 1,3 & $4,402 \pm 0,158$ & $0,816 \pm 0,077$ & $0,614 \pm 0,089$ & 3,6 & $3.030 \pm 420$ \\
\hline Amostra 3 & $1.330 \pm 130$ & 3,3 & 0,9 & $4,316 \pm 0,155$ & $0,830 \pm 0,124$ & $0,592 \pm 0,086$ & 3,8 & $2.480 \pm 370$ \\
\hline Amostra 4 & $1.150 \pm 110$ & 4,3 & 1,7 & $3,080 \pm 0,111$ & $1,268 \pm 0,156$ & $0,401 \pm 0,058$ & 4,6 & $3.730 \pm 530$ \\
\hline Amostra 5 & $1.015 \pm 105$ & 4,2 & 1,1 & $4,555 \pm 0,164$ & $0,723 \pm 0,186$ & $0,293 \pm 0,043$ & 5,4 & $4.150 \pm 630$ \\
\hline Amostra 6 & $1.020 \pm 70$ & 5,3 & 1,1 & $3,733 \pm 0,134$ & $1,151 \pm 0,080$ & $0,258 \pm 0,037$ & 7,6 & $5.200 \pm 600$ \\
\hline-
\end{tabular}

A partir dos resultados da LOE das coberturas superficiais da Superfície de Urucaia, verifica-se que as mesmas apresentaram idades entre 2.480 a 5.200 anos A.P., a 1 metro de profundidade. Neste contexto, é possível afirmar que as coberturas superficiais da Superfície de Urucaia, depositadas a 1,0 m de profundidade, apresentam em média $3.565 \pm 500$ anos A.P., ou seja, sedimentos datados do Holoceno Médio.

Considera-se a hipótese de que o processo de transporte responsável pela deposição das coberturas superficiais localizadas sobre a Superfície de Urucaia tenha sido realizado por meio de processos fluviais e eólicos durante fase climática mais seca que atual, atendendo as características texturais e morfoscópicas dos sedimentos.

Em relação aos aspectos paleoclimáticos durante o período compreendido pela deposição das coberturas superficiais da Superfície de Urucaia, considera-se que havia o predomínio de condições mais quentes e secas que as atuais, que foram responsáveis pela erosão do material de origem representado pelos arenitos da Formação Botucatu. Os mesmos em momentos de chuvas torrenciais, e devido sua baixa resistência e friabilidade, eram intemperizados e erodidos por meio da ação pluvial e eólica ao longo do Holoceno, cujo material veio a ser depositado sobre o nível localizado logo abaixo da Superfície de Cimeira, no caso a Superfície de Urucaia.

Em relação às condições climáticas, estudos realizados por Gouveia (2001), Scheel-Ybert et al. (2003) e Souza et al. (2013) comprovaram o predomínio de climas mais secos que o atual em diversos locais do 


\section{OS DESAFIOS DA GEOGRAFIA FÍSICA NA FRONTEIRA DO CONHECIMENTO \\ Instituto de Geociências - Unicamp \\ Campinas - SP \\ 28 de Junho à 02 de Julho de 2017}

Estado de São Paulo, durante o Holoceno Médio, no período correspondente à deposição das coberturas superficiais na Superfície de Urucaia.

Ferreira et al. (2013) apontam para ocorrência de atividade e formação de depósitos eólicos com atuação de clima mais seco que o atual, no vale do Rio São Francisco, em Pernambuco, durante o final do Pleistoceno Superior até a transição Pleistoceno-Holoceno, com idades entre 57.000 a 11.600 anos A.P., em um corte de uma duna com 12 metros de espessura, cujo processo iniciou-se durante o Último Máximo Glacial.

De Oliveira et al. (1999), discutem também o aumento na ativação de dunas eólicas entre 4.500 a 1.700 anos A.P. no vale do médio rio São Francisco na Bahia, corroborando com a ocorrência de processos erosivos eólicos no território brasileiro durante o Holoceno.

Assim como, Parolin e Stevaux (2006) destacam a formação de paleodunas na região Sul do Brasil, entre 4.000 a 3.000 anos A.P..

Nesta perspectiva, os dados paleoclimáticos e as análises laboratoriais corroboram com a hipótese da relação entre oscilações climáticas e deposição da cobertura superficial holocênicas na Superfície de Urucaia, durante o Holoceno.

\section{Considerações finais}

A partir dos resultados deste estudo, pode-se confirmar o papel das alterações do clima na gênese das coberturas superficiais holocênicas depositadas sobre a Superfície de Urucaia, na bacia hidrográfica do rio Corumbataí-SP.

Neste contexto, com os resultados das datações absolutas por LOE, verifica-se que os materiais das coberturas superficiais holocênicas, a 1,0 metro de profundidade, são muito recentes, datados dos últimos 5.200 anos A.P., trazendo novas contribuições na discussão sobre as interferências paleoclimáticas quaternárias na evolução da bacia hidrográfica do rio Corumbataí.

As idades obtidas são correlatas com episódios de depósitos eólicos em outros locais do território brasileiro, evidenciando ocorrência de depósitos sedimentares eólicos ao longo do Holoceno.

As condições paleoclimáticas possibilitaram a erosão do material de origem das coberturas superficiais holocênicas que recobrem a Superfície de Urucaia, a partir processos erosivos ocorridos a montante, nas áreas limítrofes da bacia hidrográfica, caracterizada pelas regiões de cimeira, formadas pelos arenitos da Formação Botucatu, que a partir de seu intemperismo depositou sedimentos no nível localizado logo 
abaixo, cujo material foi transportado pela ação fluvial e eólica, atendendo às características texturais e morfoscópicas dos sedimentos.

Logo, o uso de datações absolutas como a Luminescência Opticamente Estimulada (LOE) mostrou-se fundamental para determinar de forma precisa o momento de formação e deposição das coberturas superficiais holocênicas depositadas sobre a Superfície de Urucaia.

\section{Bibliografia}

BJÖRNBERG, A.J.S. Sedimentos pós-cretácicos do leste do Estado de São Paulo. São Carlos, 1965, 133p. (Tese de livre-docência - Escola de Engenharia de São Carlos da USP).

COMPTON, R.R. Manual of field Geology. John Wiley and Sons, New York, USA, 1962, 214 p.

CORREA, A.C.B. Dinâmica geomorfológica dos compartimentos elevados do planalto da Borborema, nordeste do Brasil. 2001, 386 p. Tese (Doutorado em Geografia) - Instituto de Geociências e Ciências Exatas, Universidade Estadual Paulista, Rio Claro, 2001.

DE OLIVEIRA, P.E.; BARRETO, A.M.F.; SUGUIO, K. Late Pleistocene/Holocene climatic and vegetational history of the Brazilian caatinga: the fossil dunes of the middle São Francisco River. Palaeogeography, Palaeoclimatology, Palaeoecology. v. 152, p. 319-337, 1999.

DULLER, G.A.T. Luminescence dating of Quaternary sediments: recent advances. Journal Quaternary Science, v. 19, p. 183-192, 2004.

EMBRAPA. Manual de métodos de análise de solo. Rio de Janeiro. $2^{a}$ ed. Atual. EMBRAPA, 1997, 212p.

FERREIRA, B.; CORREA, A.C.B.; BARRETO, A.M.F. Depósitos Eólicos Inativos do Sub-Médio São Francisco, evidências de atividade eólica durante o Pleistoceno, Pernambuco, Nordeste do Brasil. Sociedade e Natureza, v. 25, n. 2, p. 363-378, 2013.

GOUVEIA, S.E.M. Isótopos do carbono na avaliação do remonte biológico de Latossolos e Podzólicos e de eventos paleoclimáticos em distintas localidades do Brasil. São Paulo, 2001. 116f. Tese de Doutorado, Universidade de São Paulo, 2001.

INSTITUTO AGRONÔMICO DE CAMPINAS (SP). Levantamento Pedológico Semidetalhado do estado de São Paulo: Quadrícula de São Carlos. Rio de Janeiro: Aerofoto Cruzeiro, 1981. (1 mapa. Escala:1:100.000).

MELO, M.S.; PONÇANO, W.L. Gênese, distribuição e estratigrafia dos depósitos cenozoicos no Estado de São Paulo. São Paulo: IPT (IPT, Monografias 9), 1983, 74 p.

PAROLIN, M.; STEVAUX, J.C. Dry climate and eolian dune formation in the Middle Holocene in Mato Grosso do Sul state, central West Brazil. Zeitschrift für Geomorphologia. Supplementband., v. 145, p. 177-190, 2006.

PENTEADO, M.M. Geomorfologia do Setor Centro-Ocidental da Depressão Periférica Paulista. 1968, $160 \mathrm{f}$. Tese de Doutorado, Universidade Estadual Paulista, Faculdade de Filosofia, Ciências e Letras de Rio Claro. Inédito. Rio Claro, 1968.

SCHEEL-YBERT, R.; GOUVEIA, S.E.M.; PESSENDA, L.C.R.; ARAVENA, R.; COUTINHO, L.M.; BOULET, R. Holocene palaeoenvironmental evolution in the São Paulo State (Brazil), based on anthracology and soil 13C analysis. The Holocene, v. 13, p. 73-81, 2003.

SOUZA, M.M.; RICARDI-BRANCO, F.; JASPER, A.; PESSENDA, L.C.R. Evolução paleoambiental holocênica da porção nordeste do Estado de São Paulo, Brasil, 01/2013, Revista Brasileira de Paleontologia, v. 16, p. 297-308, RS, Brasil, 2013. 


$\begin{aligned} & \text { XVII Simpósio Brasileiro } \\ & \text { de Geografia Fisica Aplicada }\end{aligned}$
$\begin{aligned} & \text { I Congresso Nacional } \\ & \text { de Geografia Física }\end{aligned}$

TAVARES, A.C.; CHRISTOFOLETTI, A.L.H; SANTANA, M.P.C. Tipos de tempo e feições do escoamento superficial na Bacia do Rio Corumbataí-SP, Brasil. Climatologia e Estudos da Paisagem. Rio Claro, vol. 2 , n1. janeiro/junho, 2007, p. 128.

WINTLE, A.G.; MURRAY, A.S. A review of quartz optically stimulated luminescence characteristics and their relevance in single-aliquot regeneration dating protocols. Radiation Measurements, v. 41, p. 369-391, 2006.

\section{Agradecimentos}

Fundação de Amparo à Pesquisa do Estado de São Paulo-FAPESP. Processos: 2011/21491-7 e 2012/00145-6. 